\title{
INEQUALITIES FOR THE MIXED DISCRIMINANTS
}

\author{
CHANG-JIAN ZHAO AND XIAO-YAN LI
}

Abstract. In the paper, some new inequalities for the mixed discriminants are established, which are the matrix analogues of inequalities of the well-known mixed volumes function.

Mathematics subject classification (2010): 52A40, 26D15.

Keywords and phrases: Mixed discriminants, symmetric matrix, mixed volume, Aleksandrov's inequality.

\section{REFERENCES}

[1] M. MARCUS, I. LOPES, Inequalities for symmetric functions and Hermitian matrices, Canad J. Math., 8 (1956), 524-531.

[2] E. F. Bechenbach, R. Bellman, Inequalities, Springer-Verlag, Berlin-Göttingen, Heidelberg, 1961.

[3] H. Bergstrom, A triangle inequality for matrices, Den Elfte Skandinaviski Matematiker-kongress, Trondheim, 1949, Oslo: John Grundt Tanums Forlag, 1952.

[4] R. Bellman, Notes on matrix theory-IV: an inequality due to Bergstrom, Amer. Math. Monthly, 62 (1955), 172-173.

[5] K. FAn, Some inequalities conerning positive-definite hermitian matrices, Proc. Cambridge Phil. Soc., 51 (1955), 414-421.

[6] K. FAn, Problem 4786, Amer. Math. Monthly, 65 (1958), 289.

[7] L. Mirs Ky, Maximum principles in matrix theory, Proc. Glasgow Math. Assoc., 4 (1958), 34-37.

[8] A. Dembo, T. M. Cover AND J. A. Thomas, Information theoretic inequalities, IEEE Trans. Infor. Theory, 37 (1991), 1501-1518.

[9] A. Giannopoulos, M. Hartzoulaki And G. Paouris, On a local version of the AleksandrovFenchel inequality for the quermassintegrtals of a convex body, Proc. Amer. Math. Soc., 130 (2002), 2403-2412.

[10] M. Fradelizi, A. Giannopoulos And M. Meyer, Some inequalities about mixed volumes, Israel J. Math., 135 (2003), 157-179.

[11] X. Y. LI, G. S. LENG, Some inequalities about dual mixed volumes of star bodies, Acta Math. Sci., 25 (2005), 505-510.

[12] E. LutwAK, D. YANG AND G. ZHANG, A new affine invariant for polytopes and Schneider's projection problem, Trans. Amer. Math. Soc., 353 (2001), 1767-1779.

[13] A. D. Aleks androv, Zur Theprie der gemischten Volumina von konvexen Körpen, Die gemischten Diskriminanten und die gemischten Volumina (in Russian), Mat. Sbornik, 1938, 3, 227-252.

[14] R. SchneIder, Convex Bodies: The Brunn-Minkowski Theory, Cambridge Univ. Press, 1993.

[15] P. Pranayanuntana, Elliptic Brunn-Minkowski Theory, UMT Dissertations Publishing, 2003. 